\title{
Mechanical modeling of Lateral Cracking in Brittle Materials under Indentation
}

\author{
Ji-Peng Liu \\ Department of Mechanics, Tianjin University \\ Tianjin, China, 300072 \\ liujipeng26@126.com
}

\author{
Gan-Yun Huang \\ Department of Mechanics, Tianjin University \\ Tianjin, China, 300072 \\ g.y.huang@hotmail.com
}

\begin{abstract}
Experiments have indicated that when brittle materials are under indentation, various forms of cracks including median crack, ring crack, Hertzian crack and side (lateral) crack nucleate leading to the fragmentation. The lateral cracking under indentation can be attributed to unloading and plays a crucial role in formation of chips. Based on the mechanism of the lateral cracking, we have investigated a penny shaped crack parallel to surface under indentation with unloading. The stress intensity factors (SIFs) and the energy release rate haven been calculated. It has been found that there exists a crack size at which both SIF and the energy release rate attains maximum values. Relationship between the indentation force and that crack size has been obtained numerically. The influences of the dilatancy angle and unloading have been also demonstrated
\end{abstract}

Keywords-indentation; the lateral crack; the cavity expansion model; the sterss intensify factors; the energy release rate

\section{INTRODUCTION}

Indentation induced failure in brittle materials holds many implications in mechanical excavation, drilling and milling. Therefore, cracking in brittle materials under indentation has been investigated both experimentally and theoretically. It has been found from experiments that various forms of cracks such as Hertzian cracks, ring cracks, median cracks and lateral cracks nucleate during the indentation process $[1,2]$. Based on the elastic solution of a half space under a concentrated force, Lindquivst has analyzed [3] the stress field due to indentation. Lawn, recognizing the singularity involved in that solution, have adopted the cavity model to characterize the inelastic deformation zone under indentation and studied both the median crack and Hertzian crack problems. Scaling between the indentation force and the crack length has also been obtained which agrees well with experimental results [4].

From the perspective of material removal, the lateral cracks under indentation may play a crucial role since it has been observed that only the propagation of the lateral cracks toward the surface or their coalescence with other cracks leads to the formation of chips. Analysis of lateral cracks thus is obviously necessary. Unloading has been proposed to drive the nucleation of lateral cracks. Recent experiments on rocks and other brittle materials like ceramics have verified it [1,2]. Research on the relationship between indentation force and lateral crack length, however, is rather limited. Ahn used the sliding blister model to capture the possible inelastic deformation and considered the lateral crack problem under a sliding indenter [5]. Resorting to finite element method, Chen et al. have tackled the lateral crack problem under indentation in ceramics [6]. In their work, to capture the effect of inelastic deformation under indentation, the elastic-perfectly plastic material has been assumed to be governed by a Mises yield surface.

Since the inelastic deformation in many brittle materials is usually pressure sensitive, the Mises yield criterion is inappropriate. Consequently, we are motivated to study the lateral cracking in rocks under indentation with appropriate yield criterion, namely, Mohr-Coulomb criterion to capture the effect of inelastic deformation. The stress field due to indentation with unloading has been obtained analytically. Based on the field, the lateral crack length has been numerically related to the indentation force.

\section{MODEL AND PROBLEM FORMULATION}

To ascertain the formation and propagation of lateral crack in brittle materials under indentation, we consider the problem as shown in Figure 1 . Due to the problem with the axial symmetry, so we use the cylindrical coordinate $(\rho, \varphi, \mathrm{z})$. A spherical indenter pressed on the surface of a half space may be in the stage of unloading, which results in an inelastic deformation zone and a penny shaped crack parallel to the surface outside the inelastic zone. Since finite element analysis has demonstrated that cavity expansion model can well describe the behavior of inelastic deformation caused by the indentation [7], we will adopt it to obtain the stress field due to indentation at peak load. According to the work of Alehossein [9], the stress field due to the indentation loading can be obtained as

$$
\begin{gathered}
\sigma_{z z}^{l}=-\sigma_{r}^{*}\left(\xi_{*} / \xi\right)^{3} \cos ^{2} \theta+\frac{\sigma_{r}^{*}}{2}\left(\xi_{*} / \xi\right)^{3} \sin ^{2} \theta, \\
\sigma_{\rho z}^{l}=-\frac{\sigma_{r}^{*}}{2}\left(\xi_{*} / \xi\right)^{3} \sin 2 \theta-\frac{\sigma_{r}^{*}}{4}\left(\xi_{*} / \xi\right)^{3} \sin 2 \theta .
\end{gathered}
$$

The research reported in this paper has been supported by grant NSFC-11072701 from the National Science Foundation. 
Where $\sigma_{r}^{*}$ is radius pressure of the elastic plastic boundary, $\sigma_{r}^{*}=(2-2 \sin \varphi) /(3-\sin \varphi) q, \quad q \quad$ is the uniaxial compressive strength, $\varphi$ is the internal friction angle. The dimensionless parameters $\xi_{*}=r_{s} / a_{1}$ and $\xi=r / a_{1}$. The radius of contact with loading process $a_{1}=R \sqrt{2 \delta-\delta^{2}}$. The indentation scale $\delta=h / R$.

During unloading, the deformation is assumed to be elastic. One can write out the stress field due to unloading as

$$
\begin{gathered}
\sigma_{z z}^{u l}=-\frac{9 \Delta P(H-z)^{3}}{4 \pi^{2} a^{2}} \int_{0}^{a} \sqrt{1-(t / a)^{2}} \int_{0}^{2 \pi} \frac{1}{\rho^{\prime 5}} d \theta d t, \\
\sigma_{\rho z}^{u l}=-\frac{9 \Delta P(H-z)^{2}}{4 \pi^{2} a^{2}} \int_{0}^{a} \sqrt{1-(t / a)^{2}} \int_{0}^{2 \pi} \frac{\sqrt{\rho^{2}+t^{2}-2 \rho t \cos \theta}}{\rho^{\prime 5}} d \theta d t .
\end{gathered}
$$

Where $\rho^{\prime}=\sqrt{\rho^{2}+t^{2}+(-z+H)^{2}-2 \rho t \cos \theta}$, is the distance from the point inside contacted surface to the action point. The radius of unloading contact $a=\left[3 \Delta P R\left(1-v^{2}\right) / 4 E\right]^{1 / 3}, \Delta P$, force of unloading, $E$, the elastic modulus.

By analogy with the study on the median crack problems [4], we approximate the crack problem as superposition of the following two problems: 1) a plastically indented half space free of cracks is subjected to unloading that induces stress field $\sigma_{z z}$ and $\sigma_{\rho z}$;2) a penny shaped crack in a half space is subjected to $-\sigma_{z z}$ and $-\sigma_{\rho z}$ on the crack surfaces. Solution of the problem 2), similar to Necati's works [8], by using Hankel integral transformation, can be reduced to the following singular integral equations of Cauchy type

$$
\begin{aligned}
& -\frac{\sigma_{z}}{2 \mu}=\frac{1}{\pi} \int_{-1}^{1}\left[\frac{M}{\tau-\eta}+K_{z 2}(\tau, \eta)\right] \frac{F_{2}(\tau)}{\sqrt{1-\tau^{2}}} d \tau+\int_{-1}^{1} K_{z 1}(\tau, \eta) \frac{F_{1}(\tau)}{\sqrt{1-\tau^{2}}} d \tau, \\
& -\frac{\sigma_{\rho z}}{2 \mu}=-\frac{1}{\pi} \int_{-1}^{1}\left[\frac{M}{\tau-\eta}+K_{\rho z 1}(\tau, \eta)\right] \frac{F_{1}(\tau)}{\sqrt{1-\tau^{2}}} d \tau-\int_{-1}^{1} K_{\rho z 2}(\tau, \eta) \frac{F_{2}(\tau)}{\sqrt{1-\tau^{2}}} d \tau .
\end{aligned}
$$

Where $v 、 \mu$ are respectively Possion's ratio and shear modulus and $M=1 /(4 v-1)$. Both of $K_{z z i}$ and $K_{\rho z i}$ are the integral function respected with the first kind of Bessel function $J_{i}(i=0,1)$.

Moreover, the unknown function $F_{i}(\tau), i=0,1$, have the relationship as follow.

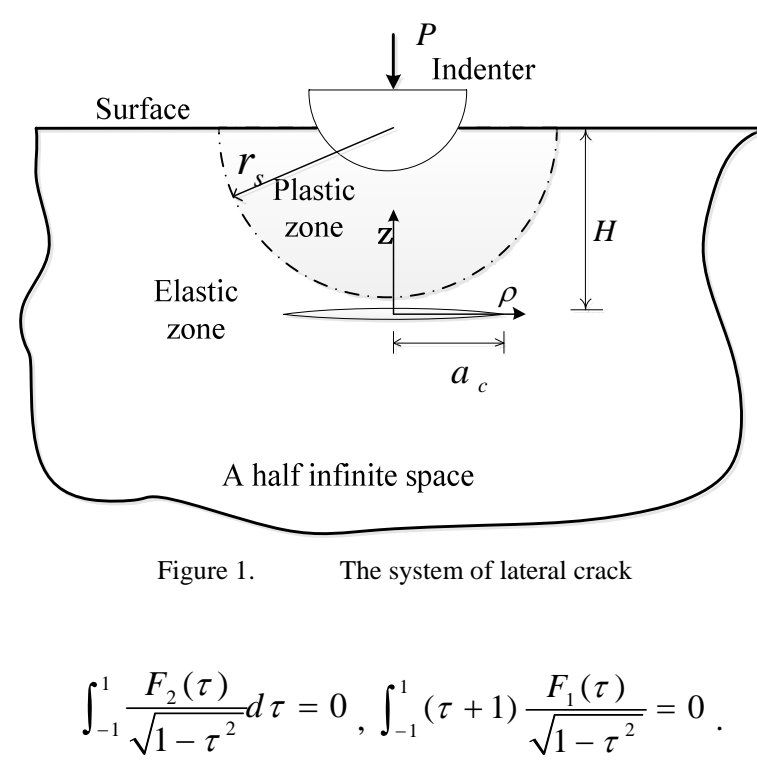

Equations (5)-(7) can be numerically solved by the discretion scheme $\left.\tau_{k}=\cos [(2 k-1) \pi / 2 n)\right], \quad \eta_{l}=\cos (l \pi / n)$, $k=1 \cdots n, l=1 \cdots n-1$. Then we obtain

$$
\begin{gathered}
-\frac{\sigma_{z}\left(\tau_{k}, \eta_{l}\right)}{2 \mu}=\sum_{k=1}^{n} \frac{1}{n} F_{2}\left(\tau_{k}\right)\left[\frac{M}{\tau_{k}-\eta_{l}}+K_{z 22}\left(\tau_{k}, \eta_{l}\right)\right]+\sum_{k=1}^{n} \frac{\pi}{n} F_{1}\left(\tau_{k}\right) K_{z 1}\left(\tau_{k}, \eta_{l}\right), \\
-\frac{\sigma_{\rho z}\left(\tau_{k}, \eta_{l}\right)}{2 \mu}=-\sum_{k=1}^{n} \frac{1}{n} F_{1}\left(\tau_{k}\right)\left[\frac{M}{\tau_{k}-\eta_{l}}+K_{\rho z 1}\left(\tau_{k}, \eta_{l}\right)\right]-\sum_{k=1}^{n} \frac{\pi}{n} F_{2}\left(\tau_{k}\right) K_{\rho z 2}\left(\tau_{k}, \eta_{l}\right), \\
\sum_{k=1}^{n} \frac{\pi}{n}\left(\tau_{k}+1\right) F_{1}\left(\tau_{k}\right)=0, \sum_{k=1}^{n} \frac{\pi}{n} F_{2}\left(\tau_{k}\right)=0
\end{gathered}
$$

The set of $n$ simultaneous algebraic equations (8)-(10) is solved and one can find $n$ values for $F_{1}\left(\tau_{i}\right)$ and $F_{2}\left(\tau_{i}\right), i=1 \cdots n$, The value of $n$ is chosen as 50 and it is adequate for the accuracy of the solution. The stress intensity factors can then be obtained by

$$
\begin{gathered}
K_{I}=\lim _{\rho \rightarrow a_{c}} \sqrt{2 \pi\left(\rho-a_{c}\right)} \sigma_{z z}(\rho, 0)=2 \mu M \sqrt{a_{c}} F_{2}(1), \\
K_{I I}=\lim _{\rho \rightarrow a_{c}} \sqrt{2 \pi\left(\rho-a_{c}\right)} \sigma_{\rho z}(\rho, 0)=-2 \mu M \sqrt{a_{c}} F_{1}(1) .
\end{gathered}
$$

And the energy release rate,

$$
G=\left(1-v^{2}\right)\left(K_{I}^{2}+K_{I I}^{2}\right) / E
$$

Obviously, the main task left is to find out the stress field $\sigma_{z z}$ and $\sigma_{\rho z}$. By superimposing the stress field of loading and unloading, $\sigma_{z z}$ and $\sigma_{\rho z}$ is obtained. 


\section{RESULTS AND DISCUSSIONS}

The parameters used for calculation are listed in Table 1. In the paper, $\delta$ is chosen as 0.1 , the depth of location of crack, $H$, is $10 \mathrm{~mm}$. For different dilatancy angles and completely unloading, we calculate the stress intensity factor as a function of crack size. In figure 2, with the increase of radius of crack, both the stress intensify factor of mode I and II first increase till maximum and then gradually decrease. This trend is mainly due to the non-uniform distribution of stress on the surface of crack. The results qualitatively agree with other models [5] and the finite element analysis [6]. Moreover, from the figure 2, we find that the different values of dilatancy angle have a great influence on the stress intensity factor, i.e., for fixed crack size, the larger the dilatancy angle, the larger the stress intensity factor. Similar effect on the energy release rate and loadindentation curve can be found that are respectively presented in figure 3 and figure 4. These results means that the von Mises criterion may fail to capture the effect of inelastic deformation on the rock fracture due to indentation.

TABLE I. THE PARAMETERS OF MECHANICS

\begin{tabular}{|c|c|c|c|c|c|}
\hline $\begin{array}{c}\text { The } \\
\text { elasti } \\
\text { c } \\
\text { modu } \\
\text { lus }\end{array}$ & $\begin{array}{c}\text { Possion, } \\
\text { s ratio }\end{array}$ & $\begin{array}{c}\text { The } \\
\text { uniaxial } \\
\text { compressiv } \\
\text { e strength }\end{array}$ & $\begin{array}{c}\text { The } \\
\text { fricatio } \\
\text { n angle }\end{array}$ & $\begin{array}{c}\text { The } \\
\text { dilatancy } \\
\text { angle }\end{array}$ & $\begin{array}{c}\text { The } \\
\text { radius } \\
\text { of } \\
\text { indent } \\
\text { er }\end{array}$ \\
\hline $\begin{array}{c}\boldsymbol{E}(\mathbf{G p} \\
\boldsymbol{a})\end{array}$ & $\boldsymbol{v}$ & $\mathbf{q ( G p a )}$ & $\boldsymbol{\varphi}$ & $\boldsymbol{\psi}$ & $\boldsymbol{R ( m \boldsymbol { m } )}$ \\
\hline 20 & 0.33 & 0.167 & $30^{\circ}$ & $10^{\circ}, 20^{\circ}$, & 5 \\
& & & $30^{\circ}$ & \\
\hline
\end{tabular}

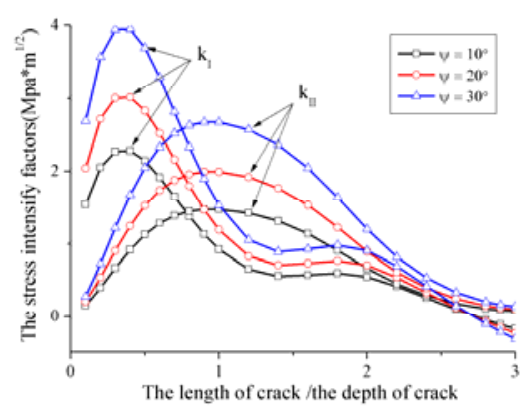

Figure 2. The stress intensity factor with the crack radius, for different dilatancy angle

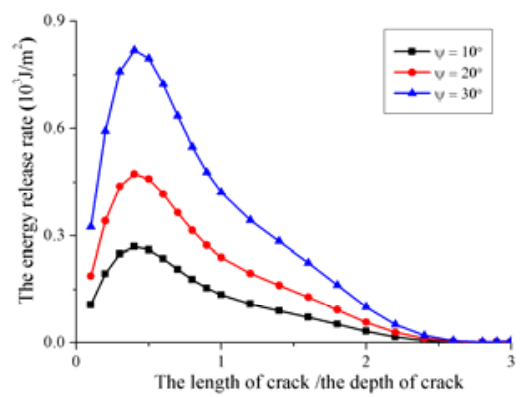

Figure 3. The energy release rate with the radius of crack, for different dilatancy angle

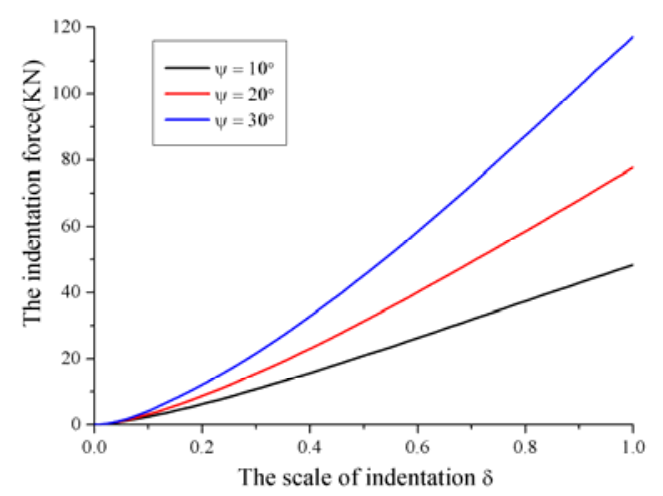

Figure 4. The load-indentation curve for different dilatancy angle

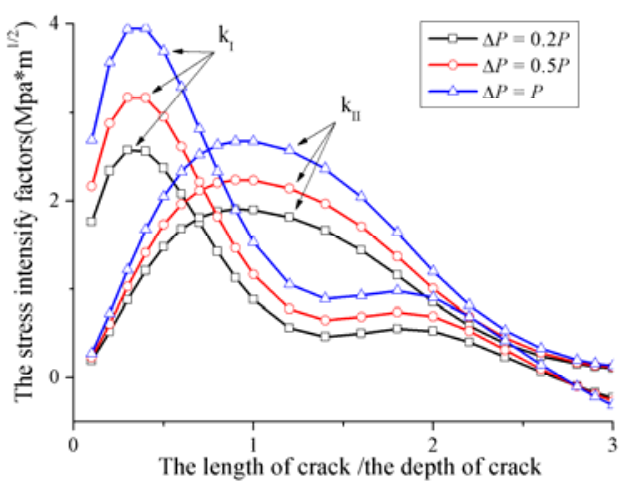

Figure 5. The stress intensity factor with the radius of crack for different levels of unloading

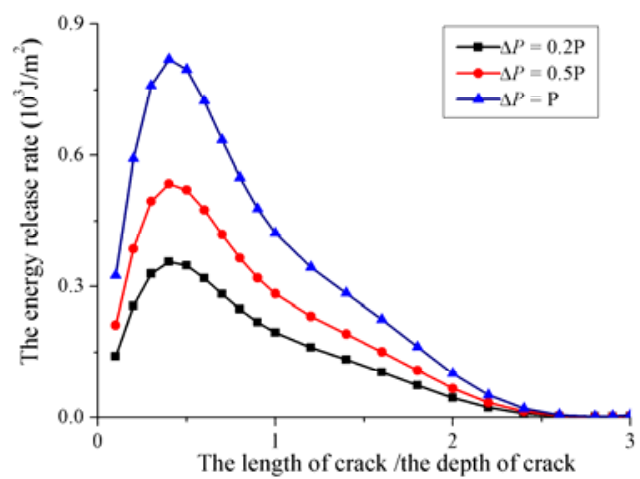

Figure 6. The energy release rate with the radius of crack for different levels of unloading

We have also calculated the stress intensity factor for different levels of unloading, with the size of indentation $\delta$ and the dilatancy angle chose to be 0.1 and $30^{\circ}$. In figure 5, we can see that the different levels of unloading have a great influence on the stress intensity factor as well, For fixed crack size, the higher the unloading level, the larger the stresss intensity factor. 
The same trend can be found for the energy release rate as shown in figure 6 .

According to fracture mechanics, when the energy release rate reaches a critical value, the cracks will propagate. In figure 6 , energy release rate achieves maximum when $a_{c} / H$ equal to 0.4. It means that for fixed indentation peak load, the crack with the length $\left(a_{c} / H \cong 0.4\right.$ ) propagates most rapidly. So the relationship of between this crack length and indentation load can be taken as the scaling for indentation induced lateral cracking.

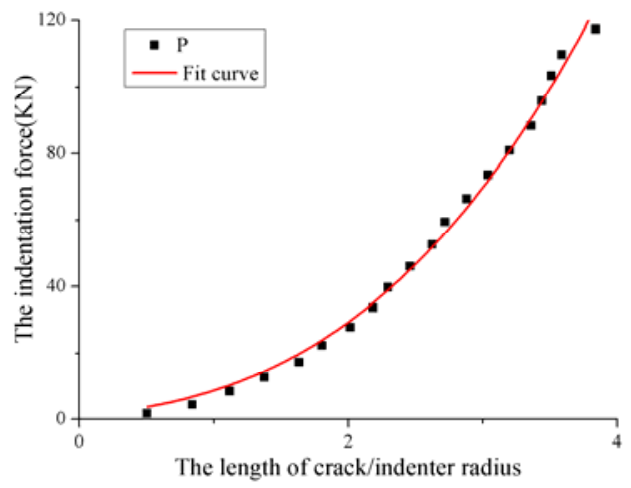

Figure 7. Relationship between the indentation force and that crack size

Accordingly, we numerically calculate the relationship between the critical length of crack and the indentation load, the result is plotted in figure 7 . The indentation load is monotonically increase with the critical length that can be optimally fit as $P=a\left(1+a_{c} / R\right)^{m}, a=1.04, m=3$.

\section{THE CONCLUSION}

In this paper, we consider the lateral cracking under spherical indenter by penny shaped crack parallel to surface.
Effects of both inelastic deformation and unloading have been captured. The stress intensity of crack tip and the energy release rate are calculated numerically. The conclusion can be summarized as follows:

1) The dilatancy angle has a great influence on the stress intensity factor and the energy release rate. Consequently, it is appropriate to use Mohr-Coulomb criteria for description of the yield of rock materials. It implies that the dilatancy of rock has great impact on the fracture by indentation.

2) The different levels of unloading have a great influence on the stress intensity factor.

3) Relationship between the indentation force and that crack size has been obtained numerically.

\section{REFERENCES}

[1] Lawn B. R., Swain M. V., Microfracture beneath point indentations in brittle solids, J. Mater. Sci., 1975, 10, 113-122

[2] Ostojic P., Mcpherson R.,A review of indentation fracture theory: its development, principles and limitations, Int. J. Fract., 1987, 33, 297-312

[3] Lindqvist P. A., Stress fields and subsurface crack propagation of single and multiple rock indentation and disc Cutting, Rock Mech. Rock Engng, 1984, 17, 97-112

[4] Lawn B. R., Fuller E. R., Equilibrium penny-like cracks in indentation fracture, J. Mater. Sci., 1975, 10, 2016-2024

[5] Ahn Y., Lateral crack in abrasive wear of brittle materials, JSME Int. J., Series A, 2003, 46, 140-144

[6] Chen X., Hutchinson J. W., The mechanics of indentation induced lateral cracking, J. Am. Ceram. Soc., 2005, 88, 1233-1238

[7] Mata, M., Casals O., Alcala J., The plastic zone size in indentation experiments: the analogy with the expansion of a spherical cavity, Int. J. Solids Struct. 2006, 43, 5994-6013

[8] Necati A., Stress intensity factors for a penny-shaped crack in a transversely isotropic layer bonded between two isotropic half spaces, Z. Angew. Math. Mech., 2003 83, 409-418

[9] Alehossein H., Detournay E., Huang H., An analytical model for the indentation of rocks by blunt tools, Rock Mech. Rock Engng, 2000, 33, 267-284 